\title{
Principio de Autonomía Deontológica para la Praxis Orientadora
}

\author{
Karina Heredia \\ kisabelhs@gmail.com \\ https://orcid.org/0000-0001-7919-2583 \\ Universidad de Carabobo \\ Puerto Cabello, Venezuela. \\ Julio González Bello \\ juliogonzalez47@gmail.com \\ https://orcid.org/0000-0001-6099-2994 \\ Universidad de Carabobo \\ Valencia, Venezuela.
}

Recibido: 25/03/2020 Aceptado: 23/05/2020

\begin{abstract}
Resumen
En estos tiempos uno de los principales problemas del profesional de la orientación se relaciona con su praxis, concretamente con los principios éticos, y específicamente con el principio de autonomía. En este sentido se puede evidenciar que el proceso de ayuda se ve influenciado por las experiencias de vida del Orientador, lo cual es una clara realidad de lo lejos que se encuentra éticamente el Orientador de su práctica. Por lo tanto, este estudio pretende crear una hermeneusis teórica del principio de autonomía deontológica del Orientador del siglo XXI a través de una metamorfosis que redefina su praxis en el medio circundante; sustentándose en la Teoría Ética Deontológica (1785) y la Teoría Crítica de la Escuela de Frankfurt (1937). Del mismo modo, este estudio se orientó en la Metodología Fenomenológico Hermenéutica de Max Van Manen (2003), el paradigma interpretativo, de nivel Interpretativo, y en el cual se develó las esencias que repercuten en la labor profesional del Orientador, encontrando el vacío teórico para fundamentar su accionar desde lo establecido en la Declaración Universal sobre Bioética y Derechos Humanos, así como también la no correspondencia del Código de ética actual con las nuevas exigencias sociales - profesionales, a su vez, esto permitió generar un postulado único que establece el principio de autonomía deontológica propio del Orientador y su medio circundante con la finalidad de garantizar la idealización del ser humano como ente capaz de generar cambios en sí mismo y en los demás, por lo que su accionar ético ha de ser cónsono con su formación académica y profesional. Al respecto no se conocen investigaciones relacionadas más que con la ética del Orientador y no con un principio que redefina su praxis haciendo de este un nuevo profesional con altas competencias éticas profesionales desde la autonomía del sujeto generador de transformaciones sociales.
\end{abstract}

Palabras Clave:Orientación, Deontología y Ética.

\section{Princípio da Autonomia Deontológica para A Práxis de Orientação}

\section{Resumo}

Nestes tempos, um dos principais problemas do conselheiro está relacionado à sua práxis, especificamente com os princípios éticos e, especificamente, com o princípio da autonomia. Nesse sentido, pode-se evidenciar que o processo de auxílio é influenciado pelas experiências 
de vida do conselheiro, que é uma realidade clara de quão longe o conselheiro é eticamente de sua prática. Portanto, este estudo tem como objetivo criar uma hermenêutica teórica do princípio da autonomia deontológica do Orientador do século XXI através de uma metamorfose que redefine sua práxis no ambiente circundante; baseado na Teoria Deontológica Ética (1785) e na Teoria Crítica da Escola de Frankfurt (1937). Da mesma forma, este estudo foi orientado na Metodologia Fenomenológica Hermenêutica de Max Van Manen (2003), o paradigma interpretativo, de nível Interpretativo, e no qual foram reveladas as essências que afetam o trabalho profissional do Conselheiro, encontrando o vácuo teórico basear suas ações nas disposições da Declaração Universal de Bioética e Direitos Humanos, bem como na não correspondência do atual Código de Ética com os novos requisitos sócioprofissionais, por sua vez, isso permitiu gerar um postulado único que estabelece o princípio Autonomia deontológica do orientador e seu entorno, a fim de garantir a idealização do ser humano como entidade capaz de gerar mudanças em si e nos outros, para que suas ações éticas sejam coerentes com sua formação acadêmica e profissional. Nesse sentido, nenhuma pesquisa relacionada à Ética do Conselheiro é conhecida, e não comum princípio que redefine sua prática, tornando-o um novo profissional com altas competências éticas profissionais a partir da autonomia do sujeito, gerando transformações sociais.

Palavras chave: Orientação, Deontologia e Ética.

\title{
Principle of Deontological Autonomy for Guiding Praxis
}

\begin{abstract}
In these times one of the main problems of the counselor is related to his praxis, specifically with the ethical principles, and specifically with the principle of autonomy. In this sense it can be evidenced that the aid process is influenced by the life experiences of the Counselor, which is a clear reality of how far the Counselor is ethically from his practice. Therefore, this study aims to create a theoretical hermeneusis of the principle of deontological autonomy of the Counselor of the 21st century through a metamorphosis that redefines its praxis in the surrounding environment; based on the Ethical Deontological Theory (1785) and the Critical Theory of the Frankfurt School (1937). In the same way, this study was oriented in the Hermeneutic Phenomenological Methodology of Max Van Manen (2003), the interpretative paradigm, of Interpretative level, and in which the essences that affect the professional work of the Counselor were revealed, finding the theoretical vacuum to base its actions from the provisions of the Universal Declaration on Bioethics and Human Rights, as well as the noncorrespondence of the current Code of Ethics with the new social - professional requirements, in turn, this allowed generating a unique postulate that establishes the principle Deontological autonomy of the Counselor and its surrounding environment in order to guarantee the idealization of the human being as an entity capable of generating changes in himself and in others, so that his ethical actions must be consistent with his academic and professional training. In this regard, no research related to the Ethics of the Counselor is known, and not with a principle that redefines his practice making this a new professional with high professional ethical competences from the autonomy of the subject generating social transformations.
\end{abstract}

Keywords: Guidance, Counseling, Deontology and Ethics. 


\section{Introducción}

Los orígenes que vinculan la Orientación con el sistema educativo nace de una acelerada industrialización que trae consigo el éxodo masivo de los campesinos a las ciudades en busca de mejorar su calidad de vida, originando cambios en el modelo educativo liberal y haciendo evidente reformas en la educación con una variedad ocupacional en los requerimientos de trabajo, encaminando así la función Orientadora que buscaba atender las necesidades de la sociedad de manera expedita.

Por su parte, la Orientación comoproceso educativo permite la integración de habilidades y competencias de los seres humanos en su pretensión de atender a la diversidad y universalizar el derecho a la educación. Así pues, esta acción debe asociarse como uno de los factores que favorecen la calidad educativa, elemento que permite la posibilidad de dar adecuadas respuestas a la pluralidad y a las necesidades de cada estudiante. Así pues, uno de los intereses más grande que presenta la Orientación en los diferentes niveles del sistema educativo venezolano, es el énfasis que se le ha dado a la práctica y al proceso focalizado en la persona que se está formando; es indudable que los problemas de Orientación en Venezuela parten de la homogenización del proceso de orientar, a lo que Vilera (2000) dice:

Debemos reconocer que hemos sido muy amplios al momento de acomodarnos a cada movimiento teórico de vanguardia en estos tiempos (autoayuda, desarrollo emocional, etc.) lo cual es un reflejo del automatismo intelectual que nos limita a concepciones inmediatistas que nos desgasta. Y con eso no vamos a lograr una identidad profesional militante, socialmente

comprometida con los cambios, con transformaciones que tengan que ver con las desigualdades sociales y la injusticia (p. 4).

Este argumento, no se aleja de la realidad que se vive en cada escenario educativo venezolano. No se investiga, no se contextualiza, se copian modelos que en lugar de solventar situaciones las agravan. Comenzando por los conflictos entre la teoría y la práctica, el aislamiento laboral, la práctica inadecuada, carencias en la supervisión, entre otras, cuestiones vinculadas a la preparación académica y profesional del Orientador.

Al respecto, González, (2001) en el Congreso Mundial de Orientación expresó: “o nos distinguimos o nos extinguimos", haciendo énfasis en la necesidad de universalizar un modelo que permitiera unificar el proceso de la Orientación como una práctica educativa 
condicionada a las necesidades propias de Latinoamérica y considerar un fundamento teórico que sustente los pilares en la cual se sostiene.

Posteriormente, Santana (2003) consideró que las cualidades que se le exigen al Orientador determinan su rol dentro del proceso e influyen poderosamente en la realización de la intervención puesto que la relación de ayuda precisa de una serie de cualidades o actitudes que este ha de transmitir al sujeto y que pueden resumirse en aceptación, comprensión y sinceridad.

Es por esto, que a la Orientación se le atribuyen diversas funciones, las cuales se encuentran establecidas en el Programa de Protección y Desarrollo Estudiantil (2007), establecido por el Ministerio del Poder Popular para la Educación como política de estado aplicable a todos los sistemas y niveles educativos.

Sin embargo, la Orientación requiere de actualización permanente respecto a nuevas prácticas, para poder abordar las diferentes situaciones que se presentan desde una visión amplia y empírica de su realidad, dado que la complejidad multidisciplinar que caracteriza el campo, obliga a los Orientadores a intervenir diferentes realidades socioculturales.

En este sentido las nuevas necesidades de la sociedad demandan el ejercicio de una práctica, de una actividad, sometida a sistematicidad, "pues la forma completamente empírica en que hasta ahora se ha llevado a cabo la Orientación, dadas las nuevas exigencias, ha dejado ser funcional” (Moreno, 2018, p. 68).

Asimismo, González (2008) expresa que:

La Orientación y la educación han sido consideradas como procesos complementarios donde la práctica de la primera debe incidir en los niveles de calidad de la segunda. Definitivamente la posibilidad de la no extinción de la Orientación depende de la capacidad de desarrollar nuevas prácticas para las nuevas dimensiones de la educación del siglo XXI (González, 2008, p. 90).

Por lo tanto, uno de los retos que debe afrontar la Orientación es sin duda, hacer de la práctica un hecho más real respetando las diferencias individuales, sociales y culturales en el proceso de ayudar a otros, ya que la Orientación que se ha venido ejerciendo no ha beneficiado a todas las personas que de alguna manera la han requerido, consecuencia de la falta de conciencia de algunos profesionales del área.

A propósito de ello, se puede observar la realidad vista en el Estado Carabobo Venezuela, el cual es uno de los dos estados que egresa a nivel universitario Orientadores en el 
país, por lo que Heredia (2017) a través de una investigación aplicada al 30 por ciento de los Orientadores de diferentes Municipios, en torno a la interpretación y la puesta en práctica de la Orientación en el campo laboral, determinó que el 12 por ciento de los encuestados al no tener claro cómo, por qué y para qué orientar, buscan reproducir lo que a su vez otros Orientadores realizan en la cotidianidad, teniendo que enfrentar de forma empírica los retos de su práctica.

Lo que quiere decir, que existe un evidente divorcio entre la teoría y la práctica, específicamente con el principio de autonomía tomando en cuenta que este es concreto con respecto a su utilidad en el campo laboral. Por consiguiente, fue necesario precisar que el profesional de la Orientación cumple sus funciones, solo cuando posee criterio suficiente para entender los problemas sociales y educativos y da respuestas curriculares de calidad a las situaciones y espacios educativos que se le presenten.

\section{Escenario de la Investigación}

A la Orientación se le han formulado algunos reclamos relacionados con la efectividad y eficacia en su actuación y que justifican la búsqueda de la re-conceptualización (González, 2008). Por lo que el Orientador, ha tenido que resguardarse en diferentes métodos para dar respuesta, siendo una situación de interés y necesidad peculiar que proviene de muchos autores de diversas latitudes, por lo que muchas investigaciones buscan redefinir la teoría y reconceptualizar su praxis. Es así, como en la praxis de la Orientación se debe asumir el compromiso ético de promover la alteridad, fomentar el respeto y la aceptación de las diferencias, como condiciones necesarias convivencia democrática, inclusiva y consiente del derecho de las personas para el logro de la justicia social traducida en oportunidades para todos, partiendo de un enfoque social, comprometido con él mismo, sin obviar al otro, en el respeto de los derechos humanos, para alcanzar la tan ambicionada igualdad. (Camargo y Leal, 2015)

Por consiguiente, la inquietud por una praxis acorde con las demandas sociales, está calando en numerosos estudios de diferente amplitud geográfica, explorando en ellos las necesidades de formación que emplazan con más frecuencia a los profesionales de la Orientación, de acuerdo con competencias profesionales que se perciben como necesarias y los aspectos éticos claves en el pleno desarrollo de sus facultades profesionales.

En el mismo orden de ideas la Asociación Internacional para Orientación Educativa y Vocacional (IAEVG) a través de la IOEP establece como norma ética de sus miembros, ser 
conscientes de sus valores y actitudes, para ser discretos y objetivos en la asistencia que proporcionan a sus clientes. En concreto, evitan toda forma de discriminación y de estereotipo racial, sexual y de edad. De la misma manera que, los casos en los que las cuestiones éticas sean ambiguas o no sean suficientemente claras, buscarán el asesoramiento o consulta confidencial de una asociación profesional o de un colega para intentar clarificar la cuestión o desarrollar estrategias para rectificar las condiciones que causaron el problema, siendo un ejemplo más claro y preciso de lo que un Orientador en la más simple de sus funciones debe desarrollar para demostrar calidad educativa y curricular en su ejercicio profesional.

Por consiguiente, toda profesión debe disponer de una ética que regule y conforme el “deber ser" de todo aquello que en ella se trate, por lo que se presenta el código deontológico, el cual es el conjunto de normas que regulan el ideal de las relaciones humanas, ya que la dimensión ética tiene sentido "cuando entra en escena el otro" (Eco, 1998). En este sentido, se convierte en una demanda de toda profesión.

En consecuencia, existen profesiones que por su vinculación a las personas, exigen el desarrollo de una ética explícita: entre ellas encontramos las profesiones educativas y de manera especial las relacionadas con el campo de la Orientación.

Por lo que se hizo necesario, considerar la bioética que "nace como una actuación multidisciplinaria de los científicos por dominar y transformar la naturaleza, logrando un dominio y una capacidad de transformación del mismo ser humano"; y que además, posee sus cimientos en la Declaración Universal sobre Bioética y Derechos Humanos adoptada por la Conferencia General de la UNESCO en 2005 en la que tratan "las cuestiones éticas relacionadas con las ciencias de la vida y las tecnologías conexas aplicadas a los seres humanos, teniendo en cuenta sus dimensiones sociales, jurídicas y ambientales".(p.86)

Por lo tanto, los principios éticos deontológicos que se sustentan en la bioética, cubren un conjunto de investigaciones, discursos y prácticas, generalmente pluridisciplinarias, que tienen como objeto aclarar y, si es posible, resolver preguntas de tipo ético, suscitadas por la investigación en el seno de sociedades caracterizadas, en diversos grados, por ser individualistas, multiculturales y evolutivas.

En este sentido, aunque los principios éticos son fundamentales, se limitan entre si ya que en su aplicación a una situación concreta se requiere del buen juicio, es decir, de una 
ponderación adecuada por parte de quien tiene que tomar la decisión, y esta ponderación exige tomar en serio los derechos e intereses de los otros. (Amaya y Otros, 2015).

Es necesario, por tanto, establecer pautas de actuación fundamentadas en los códigos deontológicos y las normas éticas que se imponen desde la tarea educativa. A su vez, la competencia profesional es también una cuestión ética. Se consideran contrarias a la competencia profesional las malas prácticas como por ejemplo el uso de técnicas o instrumentos no contrastados científicamente o la prolongación de las intervenciones sabiendo que no son eficaces. Por lo que va contra la ética profesional el uso de procedimientos ajenos a la Orientación o aquellos para los que los Orientadores y las Orientadoras no estén cualificados.

Por lo tanto, el profesional de la Orientación debe saber cuándo las necesidades del orientando demandan otros profesionales y no extralimitarse de sus funciones. Por ello, se hizo necesario considerar los Principios Universales de la Ética y los subyacentes códigos deontológicos de la Psicología y la Orientación en los que se reflejan: la no maleficencia que considera la obligación a no hacer daño o mal intencionadamente, de prevenirlos, evitarlos o rechazarlos y de hacer o promover el bien. Seguidamente, el principio de Beneficencia, el cual es la adjudicación de beneficios, el análisis perjuicio beneficio y costo beneficio, esta contribuye con el bienestar de ayudar a las personas de manera activa.

Igualmente el principio de justicia, que considera las normas que garantizan la distribución justa de beneficios, busca solidaridad social, mediante la distribución igual, equitativa y apropiada no solo de bienes materiales, sino de derechos y responsabilidades en la sociedad, para evitar discriminaciones e injusticias y promover la aplicación de lo correcto y debido y finalmente el principio de autonomía que está unida con la libertad de acción independiente de influencias y con la capacidad de actuar intencionadamente que es donde se centra con especial interés esta investigación, puesto que el principio de autonomía obliga a los profesionales a procurar y asegurarse de la comprensión de la información técnica que ofrecen y defender la voluntariedad de las personas, grupos o comunidades en la toma de decisiones.

A juicio de Rubio (1992), la autonomía es uno de los valores morales en general, no es una capacidad individual, no es un estado o atributo de las personas, sino un ejercicio, una cualidad de la vida que viven. Se tiene que hablar, por tanto, de procesos y situaciones sociales 
en las que las personas se conducen automáticamente y, en ese proceso, construyen su identidad ética

En atención a lo que se viene planteando, el principio de autonomía es al mismo tiempo un derecho y una capacidad. Como derecho hace referencia a la potestad que tienen las personas para decidir sobre sus propios asuntos y en especial sobre su propio cuerpo. La autonomía como derecho va en contravía del paternalismo y por ello exige a los profesionales no intervenir sin el consentimiento de la persona sujeto de atención, así dicha intervención sea en su beneficio. (Amaya, Ob.cit)

Como capacidad, la autonomía tiene que ver tanto con las habilidades intelectuales y afectivas del usuario como también de las oportunidades para ejercer dicha autonomía. Una persona que por diversas circunstancias tiene limitaciones para llevar a cabo deliberaciones racionales, no tiene la capacidad para ejercer su propio juicio y en este sentido no es capaz de ser autónoma, permanente o transitoriamente, según sea el caso. En otras palabras, la autonomía es reconocida como el derecho que tienen las personas competentes a ejercer la regulación personal de forma libre y sin interferencias externas que impidan ejercer sus elecciones. De esta forma, los individuos actuarán de forma intencional, con conocimiento de sus elecciones y ausentes de influencias externas que pretendan controlar y determinar el acto. (Amaya y otros, 2015)

Sin embargo, las contradicciones entre la autonomía humana y la práctica de la autonomía, lleva a revisar lo que plantea Festinger acerca de la teoría de la Disonancia Cognitiva, citado por Vadillo (2004, p.17) "las personas no soportamos mantener al mismo tiempo dos pensamientos o creencias contradictorias, y automáticamente, justificamos dicha contradicción, aunque para ello sea necesario recurrir a argumentaciones absurdas", lo que quiere decir que el ser humano necesita siempre sentir que todas sus acciones, pensamientos y creencias son coherentes.

Como consecuencia de ello, los conceptos que a lo largo de toda la vida se han venido construyendo y reforzando con los pensamientos incongruentes que se traducen en actos y prácticas formativas contradictorias en el deber como formadores.

Por lo tanto, tener derecho a la autonomía no significa elegir al antojo, significa que para elegir no sólo nos guiamos por el tribunal de nuestras conciencias, sino que es necesario asumir en lo posible una perspectiva imparcial de juicio y reconocer la igual libertad de los 
demás. Así pues el respeto a la autonomía, establece la necesidad de respetar la capacidad de las personas para tomar decisiones. Es la regulación personal, libre de interferencias externas y limitaciones personales que impiden hacer una elección. Significa simplemente que se va a actuar de una forma ética y moral, se elegir por sí mismo lo que se va hacer. Por supuesto, se pueden recibir sugerencias o persuasiones que provienen de fuentes externas, pero a la hora de la verdad, se debe elegir por sí mismo. Es solo entonces cuando aquello que se ha hecho se puede imputar, por lo tanto es un acto propio, de tal modo que se es responsable, digno de elogio o culpa.

Sin embargo, hoy por hoy, una de las dificultades presentadas respecto al principio autónomo de los Orientadores, viene dada por la falta de definición de un perfil profesional y la asignación de un número de funciones lo que a su vez se ve reflejado en la forma que realizan su práctica. (Luque, 2005),

A modo de entender los diversos planteamientos, la situación de crisis de la Orientación en los momentos actuales está dominada por una gran paradoja, la cual se expresa en el hecho de que si bien hay consenso en justificar la práctica de la Orientación, las necesidades-carencia han ido en aumento $y$, en consecuencia se ha incrementado la discrepancia entre lo dado y lo deseable, por lo que la Orientación se ha ido debilitando. (Casado, 2010)

Como resultado, se presenta a la Orientación como una práctica social que muestra una falta de especificidad en su ámbito de acción, pues históricamente ha tenido que responder a necesidades de índole muy diversa.

Con mayor peso en la contextualización del problema, se tiene que durante el transcurrir de los años, se han profundizado cambios en el paradigma educativo, y por consiguiente en el modelo de Orientación adoptado, teniendo entonces los profesionales en esta área, adaptarse a diferentes tipos, niveles y experiencias de formación, lo cual conduce a que interpreten su función Orientadora de modo diverso, que es para lo cual se centra el nudo crítico de la investigación, el cual tiene por finalidad hacer una hermeneusis del principio de autonomía que manejan los Orientadores y como a través de este principio ejecutan su praxis, pretendiendo lograr así una metamorfosis de este principio, es decir, ir más allá de la forma inicial para dar respuesta a una sociedad en constante cambio. 
Al respecto, en esta nueva era se necesita que la Orientación, genere respuestas de su rol, vinculado con la aplicación de recursos propios para un ambiente de aprendizaje e investigación que establezca su pertinencia social con las exigencias sociales actuales y de desarrollo científico en Venezuela, por lo que de acuerdo a las demandas socio-históricas de la Orientación revisadas durante este proceso investigativo emergieron las siguientes interrogantes: ¿De qué manera el Orientador debe abordar el principio de autonomía deontológica desde su praxis? ¿Cuáles efectos sociales colaterales acarrea el desconocimiento por parte del Orientador del principio de autonomía establecido? ¿Qué implicaciones morales lleva al Orientador a no ejecutar su praxis conforme a lo curricularmente establecido? ¿Qué cambios o metamorfosis debe sufrir el principio de autonomía para poder adaptarse a los nuevos tiempos y sus exigencias?

Por consiguiente el propósito de la investigación estuvo centrado en construir una hermeneusis teórica del principio de autonomía deontológica del Orientador del siglo XXI a través de una metamorfosis que redefina su praxis en el medio circundante.

\section{Método}

En esta investigación, se evidencia la metodología impulsada por Max Van Manen (2003), que parte del hecho de que no hay método, sino un conjunto de procedimientos (p. 48). Por lo que fue el proceso utilizado en el desarrollo de este estudio, dado que no condiciona el constructo investigativo a una forma específica para descubrir las esencias que emergen del contexto.

Por tanto, se trata del estudio de un todo integrado que forma o constituye una unidad de análisis y que hace que algo sea lo que es: una persona, una entidad étnica, social, empresarial, un producto determinado, etc.; donde, se tenga en cuenta los nexos y relaciones que tiene con el todo, los cuales contribuyen a darle su significación propia.

De esta manera, esta investigación respondió al enfoque cualitativo, procurando la identificación de la naturaleza profunda de las realidades, su estructura dinámica, aquella que da razón plena de su comportamiento y manifestaciones.

Así pues, el propósito no es generalizar conocimientos, probar hipótesis, elaborar leyes, establecer relaciones entre variables, etc. Su contribución consiste en la profundización de los significados esenciales de la experiencia en Orientación. A nivel formal, el investigador llega a elaborar "una descripción (textual) estimulante y evocativa de las acciones, conductas, 
intenciones y experiencias humanas tal como las conocemos en el mundo de la vida" (Van Manen, Ob. Cit. p.37).

En todo caso, la fenomenología, es la descripción de la cualidad viva de la experiencia vivida y, por el otro, también es la descripción del significado "de las expresiones" de la experiencia vivida. Los dos tipos de descripción parecen en cierto sentido diferentes, puesto que la primera es una descripción inmediata del mundo d la vida tal como es vivido, mientras que la segunda es una descripción intermediada o mediada del mido de la vía tal como se expresa en forma simbólica. Por lo que hay quienes sostienen que toda descripción es en última instancia una mera interpretación. "El significado de la descripción fenomenológica en tanto que método reside en la interpretación (...). La fenomenología es hermenéutica en el sentido primordial de esta palabra, donde se designa esta actividad de interpretar. (Heidegger)" (1962, p.37)

En este punto, los investigadores tratan de entablar relaciones estrechas y abiertas con alguno de los individuos del escenario de investigación. De esta manera, en función del presente estudio, los informantes claves estuvieron conformados por Orientadores en ejercicio libre y dependiente ubicados en el Estado Carabobo, pertenecientes a diferentes estratos educativos.

Algunos de los métodos empíricos propuestos por Van Manen (2003) son: la descripción de experiencias personales, las experiencias de otros u obtención de descripciones en fuentes literarias); la entrevista conversacional, y la observación de cerca. Cada una de estas actividades, en apariencia comunes a otros enfoques de investigación de corte cualitativo, tienen como peculiaridad fundamental la naturaleza de la pregunta que se formulan.

Para efectos de la presente investigación realizó la descripción de experiencias personales y la entrevista conversacional. Por su parte, la descripción de experiencias aspira que las descripciones obtenidas estén libres de interpretaciones causales, explicaciones teóricas, detalles circunstanciales poco relevantes y valoraciones subjetivas, asimismo, la entrevista conversacional, aspira a obtener el significado vivido de determinada experiencia prescindiendo de las interpretaciones subjetivas acerca de ella. (Van Manen, Ob. Cit)

En el desarrollo de la investigación actuaron transversalmente dos impulsos metodológicos fundamentales: la reductio o reducción y la vocatio o dimensión vocativa. La 
reducción es la actitud de fondo y la forma que adopta la reflexión para llegar a captar las estructuras esenciales de la experiencia y que se inspira en el método de la reducción ideado por Husserl.

Por su parte, Van Manen sugiere diferentes modalidades de reducción susceptibles de ser aplicadas de forma simultánea y/o secuencial en la investigación. Sin embargo para efectos de la presente investigación, se tomará en cuenta la reducción eidética.

Al referirnos a la Reducción Eidética es perentorio mencionar el Diccionario de la Real Academia Española (RAE) que define el término de eidético como aquello que guarda relación con los conocimientos. Este término es de origen griego "cĩ $\delta o \varsigma$ ” o eidos, o sea "forma" que se emplea en el ámbito filosófico para describir lo relacionado a la esencia. Por lo tanto, puede decirse que lo eidético es un concepto opuesto a lo fáctico y a lo sensible, esta palabra también abarca la idea de que el individuo que es capaz de proyectar imágenes o vivencias con anticipación; por lo que también es considerado como la capacidad que tienen algunas personas de recordar tanto cosas que han visto como que han oído anteriormente, en algún momento de sus vidas.

Respectivamente, la palabra eidético, es empleada en el ámbito de la fenomenología para describir a aquellas acciones en las que un ser humano en concreto sólo recuerda lo más importante, las principales señas de identidad. Por lo que la reducción eidética es un proceso que se basa en la eliminación de cualquier clase de posición que se adopte frente a la realidad de una vivencia y de su objeto, así como también de cualquier postura vinculada a la naturaleza de los sujetos.

Asimismo, este método está relacionado a la convicción de que la mente humana es como una especie de tabla rasa, que carece del sentido de conciencia previo a su adquisición por medio de la experiencia, por lo que la reducción eidética consiste en apartar o excluir todo lo que no es dado en la pura esencia del fenómeno.

Por su parte, la vocatio recoge el conjunto de estrategias de escritura que usa el investigador para revelar de forma convincente, mediante un texto fenomenológico la naturaleza y la importancia del fenómeno estudiado.

A continuación, se describen las fases del Método de Van Manen que contempla el contenido y las actividades a ejecutar como parte de la metódica impulsada por este autor, 
próximamente se especifican cada una de estas a modo de comprensión de cómo se abordará la realidad vivida en pleno contexto de los informantes clave y el investigador.

\section{Descripción la experiencia vivida}

En esta etapa de naturaleza más descriptiva se integra el proceso de recogida de la experiencia vivida desde fuentes diversas: relatos de la experiencia personal, protocolos de la experiencia y entrevistas conversacionales

Se inicia la investigación con la escritura de historias personales acerca de la experiencia en Orientación. A continuación, se solicita la escritura de anécdotas relacionadas con el principio de autonomía deontológica. En efecto, lo que se aspira que las descripciones obtenidas estén libres de interpretaciones causales, explicaciones teóricas, detalles circunstanciales poco relevantes y valoraciones subjetivas. Por este motivo, la escritura de protocolos por parte de los participantes debe ser adecuadamente orientado. Por lo que en las recomendaciones se les pide describir la experiencia tal como ha sido vivida, evitando describir explicaciones causales (como por ejemplo: "yo creo que esto se debe a..."), generalizaciones ("supongo que por eso...") o interpretaciones abstractas (“como se sabe..."). Se describe por tanto, la experiencia “desde dentro": lo que se piensa y sentía en ese momento.

Un segundo momento estuvo orientado a la ampliación y reescritura de anécdotas. En una nueva entrevista se intenta constatar la "fidelidad" del relato a la experiencia vivida por las personas y profundizar en la misma. Para la obtención de contenido relevante y responder las

cuestiones fundamentales (¿Cómo es la experiencia práctica del Orientador?, ¿Cuál es su valor desde el principio de la autonomía deontológica?), se formula una guía de preguntas a partir del análisis de cada protocolo. A partir de lo obtenido, se reformulan la anécdotas integrando lo más relevante desde el punto de vista del significado "vivido" descartando lo accesorio. En todo momento se procura mantener la fidelidad a la experiencia de la persona, usando sus mismas palabras y el sentido que a ellas les dio.

\section{Interpretación}

En esta etapa el esfuerzo se concentró en la reflexión e interpretación del material experiencial. Para ello, se realizó el análisis temático. (Ayala, 2007, pp.171-175). En el nivel más general de este análisis (reflexión macro-temática) se detecta la frase sentenciosa que 
pretende captar el significado fundamental o la importancia del texto como un todo (aproximación holística y sentenciosa). (Van Manen, 2003, pp.108-113).

En el siguiente nivel (reflexión micro-temática) se aplicó la aproximación selectiva o de marcaje y la aproximación detallada o línea a línea para obtener un conjunto de frases que “capturaran”, por así decirlo, los significados esenciales de la experiencia.

A medida que se desenvuelve este análisis, se verificó la redacción de transformaciones lingüísticas. Esto significa recoger en párrafos más sensibles, desde el punto de vista fenomenológico, los temas y afirmaciones temáticas reunidas hasta el momento. Es importante mencionar que "redactar transformaciones lingüísticas no es un procedimiento mecánico: se trata más bien de un proceso hermenéutico y creativo" (Van Manen, 2003, p. 113).

Una vez extraídos los significados, es necesario cuestionarlos a fondo: ¿Es "esto" realmente un rasgo esencial de la experiencia en Orientación? Para responder a esto, se lleva a cabo la reducción eidética con cada una de las afirmaciones hechas en el análisis temático. Mediante el método de la variación imaginativa, se verificará la relación esencial o accidental del tema con el principio de deontología. Se trata de realizar un esfuerzo imaginativo de supresión hipotética de un tema que aparenta ser esencial para el ser del fenómeno mismo. (Van Manen, 2003 p.125)

Una vez explicitadas, estas ideas se sometió a una autoconciencia crítica que descubre su naturaleza ideológica. De esta forma se consigue una apertura genuina al fenómeno objeto de estudio.

\section{Descripción - Interpretación}

Durante el proceso reflexivo y, de forma intensiva, se redactó el texto fenomenológico recogiendo los hallazgos de la investigación. El objetivo de la investigación fenomenológicohermenéutica desde la perspectiva de Van Manen es el de "elaborar una descripción (textual) estimulante y evocativa de las acciones, conductas, intenciones y experiencias humanas tal como las conocemos en el mundo de la vida" (Van Manen, 2003, p. 37).

Este texto, debe ser capaz de expresar tal mismo tiempo significado de tipo cognitivo o expositivo y significado no cognitivo. En el primer caso "se trata de las significaciones semánticas de las palabras y discursos en el habla y la escritura y en el segundo caso se refiere a la cualidad expresiva de los textos". (Van Manen, 2003 p. 19) 
Con el texto fenomenológico se pretende provocar en el lector, un efecto transformativo de forma que su significado más profundo haga una evocación edificante al yo del lector motivando así a la repentina percepción de una comprensión intuitiva del significado vivido de algo.

\section{Teorización}

La naturaleza de la experiencia educativa explica por qué para el estudio de ciertos fenómenos resulta inadecuada la opción de enfoques de investigación centrados en la obtención y el desarrollo de conocimiento de naturaleza lógico-formal y/o técnica. Por ende, es necesario hallar vías de investigación y teorización adecuadas para aprehender y desarrollar conocimiento relevante sobre los aspectos esenciales de la experiencia pedagógica.

En este sentido, la Fenomenología Hermenéutica constituye una aproximación rigurosa y especialmente fructífera para alcanzar este objetivo. En realidad, únicamente la Fenomenología Hermenéutica ofrece la posibilidad de adentrarnos en la naturaleza normativa, pática, prerreflexiva, situacional y conversacional de esta experiencia humana. De esta forma, como enfoque orientado a las dimensiones más personales y humanas de la educación complementa los enfoques metodológicos más orientados al desarrollo de la dimensión técnica de la misma. En este sentido, responde a "la necesidad de que la investigación educativa consolide, desde un pluralismo metodológico, una forma de construir las ciencias pedagógicas que implique una mejora real para la educación y, por ende, al desarrollo humano" (Bartolomé, 2000, p. 22).

Desde una perspectiva más amplia, la Fenomenología Hermenéutica desde la aproximación de Van Manen pretende ser una respuesta para algunas deficiencias que afectan a la teorización educativa en general.

Por su parte, la Fenomenología Hermenéutica asume como punto de partida y de llegada de la investigación el mundo de la vida. Este mundo de la vida es "el mundo tal como lo experimentamos inmediatamente de un modo prerreflexivo, y no tal como lo conceptualizamos o categorizamos, ni como reflexionamos sobre él” (Van Manen, 2003, p. 27). Del mismo modo, aspira a responder a otra exigencia crucial de la educación: desarrollar conocimiento pertinente que capacite al educador para afrontar lo particular y único de las situaciones educativas vividas. Para ello, se propone desarrollar la teoría de lo único, es decir, un conocimiento que posibilite afrontar la unicidad de cada experiencia a la que se enfrenta el 
Orientador. Como explica Van Manen: “(...) la fenomenología es, en un sentido amplio, una filosofía o 'teoría de lo único'; se interesa por lo que es en esencia irremplazable” (Ob. cit. 25).

\section{Apertura de la autoconciencia crítica en el proceso de reflexión de la experiencia vivida.}

El arte que permite develar el fenómeno, es una poesía simple en la historia del investigador y su habilidad para descubrir de forma genuina el problema visto no solo desde su perspectiva, sino también desde la otredad en la concreción de la persona como individuo diferente, que siendo parte de un contexto, reconoce su existencia y su poder evocativo de hacer y ser parte de la historia. Así es como el Orientador en la majestuosidad de su labor profesionalmente ética - es y debe ser capaz de actuar. Es la sinfonía que le permite acoplar la teoría y la práctica, es hacerla suya en un ser y permitirse vivir y experimentar la historia desde la alteridad.

Tal como se muestra en la experiencia que se narra en la Anécdota 01, es indiscutible negar que el primer encuentro es punta de lanza que permite entrelazar la historia y las condiciones que llevan a este profesional a actuar de un modo específico. Mientras más acoplado a la historia del otro esté, mayor será su capacidad de entender y comprender las circunstancias que conducen a las personas a actuar de una forma específica.

Ciertamente es la escucha activa, el medio ideal para llegar a entender el problema visto desde el otro, es la lucha en contra de les expectativas y justificar que el fin conduce a los medios. En esta experiencia, se aprecia como el Orientador supone que quien recibe la ayuda debe estar al tanto de las competencias para las cuales está capacitado y es una vía obligada a tener que dar la información sólo porque este, dentro de una institución está capacitado para tal fin.

Es en el proceso de relación de ayuda, donde los individuos se conocen y el establecimiento de la confianza se da por la capacidad del profesional de hacer valer sus competencias, habilidades y destrezas; es permitir que el proceso llegue a la persona como una vía para entender el problema y buscar dentro de sí lo más conveniente.

Es por ello que en la experiencia 02 se hace visible la necesidad de separar las condiciones, estructuras, valores, pensamientos y paradigmas del proceso de relación de ayuda, para mantener una posición libre de prejuicios. Conducir una entrevista y condicionarla al componente religioso del Orientador es irrespetar la individualidad, los 
valores y las creencias del otro, derrumbando cualquier condición adquirida desde la niñez hasta la vida adulta, es la sublevación del más alto nivel de pertinencia arraigado hacia la familia. Situaciones como estas, ponen en tela de juicio la capacidad del Orientador de utilizar sus herramientas profesionales, dejando la situación o el problema a favor de cualquier posición personal, bien sea ortodoxa o no ortodoxa, partidista o adoctrinada a una formación distinta al otro, es el irrespeto a la formación y las estructuras curriculares establecidas en la vida universitaria.

De igual manera, la experiencia 03, es una evidencia de que estimar las acciones futuras o permitirse creer lo que ha sucedido antes de que la persona pueda terminar su oratoria, ocasiona formarse estructuras mentales o falsos positivos de lo que pudiera estar generando un comportamiento. Peor es aun, actuar en base a este pensamiento y luego darse cuenta que las cosas no eran como se pensaban, esto trae confusión, sensación de haber perdido el tiempo, frustración por ofrecer alternativas erradas a una situación no expuesta y conseguirse que no hay evolución del problema porque sencillamente la solución era para otro tipo de esquema.

En continuidad, más grave es la situación como se presenta en la narración de la experiencia 05 donde el Orientador se auto invita a ser parte del proceso, en buscar en nombre de otros las alternativas que necesita para cambiar y peor aún permitirse sentirse frustrado porque la persona no cumple con la estructura mental que este posee. No es posible éticamente, permitirse engancharse con las situaciones del otro ya que eso hace más difuso el problema.

Por ello, siempre la práctica vista desde la teoría, será la mejor alternativa para dar respuestas idóneas a cada situación, actitud que se pudo observar en la narración de la experiencia 07 , donde al mantener la neutralidad existe mayor capacidad de saber que siempre se puede ser útil., cualidad que no debe ser puesta en duda ni puede ser una sorpresa al maravillarse con la resolución de un problema, y aquí se generan varias interrogantes, siendo una de ellas: ¿Si estoy sorprendido por haber ayudado a resolver un problema, qué pudo haber sucedido con las situaciones anteriores? ¿Quizás no manejaba el tema, el contexto? ¿O mis creencias personales no me permitieron dar una respuesta oportuna? ¿Quizás el principio de autonomía deontológica es una vía idónea para conocer si realmente estoy en lo correcto? 
Esto, fue lo que se demostró con la experiencia 09, que permitió denotar que accionar eficazmente ante una situación, es la garantía de lo preparado que se encuentra profesionalmente un Orientador, “...contar con las herramientas profesionales, tener conocimiento de las redes de apoyo y saber qué hacer en el momento precioso es la mejor demostración de un profesional que sabe lo que hace..." y esto me lleva a generar otra interrogante, ¿qué es entonces lo que hace que unos Orientadores sean más eficientes que otros? ¿Es su condición social?, ¿Es su preparación académica?, ¿Es un rasgo que incide en su situación familiar? o es acaso el conocimiento de su perfil profesional y el respeto a los más altos niveles de conciencia donde se evidencia el principio de autonomía deontológica, que si bien, ha sido generalizado, es necesario pensar en especificar su descripción conceptual a otra estructura cognitiva y apropiarse de su significado como la herramienta madre que permite a los Orientadores actuar con solidez y pertinencia.

Así pues, es en la transformación de las estructuras cognitivas, la adecuación y contextualización del presente siglo, que deben perfilarse las investigaciones científicas; es ahondar en el problema, descubrir el fenómeno y comprender, así como ser capaz de dar respuestas, evolucionar con las necesidades, anticiparse suspicazmente, ello implica un esfuerzo enorme en querer cambiar, darse cuenta y corregir, entender que las generalizaciones de los preceptos que hoy en día se conocen como correctos necesitan ser reformulados, porque las situaciones pasadas no son ni medianamente las mismas, el ser humano girará, se perderá y se encontrará como un círculo vicioso si las aristas que tenemos en las manos por resolver no son expuestas al mundo como un llamado a cambiar e ir más allá de la forma, es una metamorfosis del conocimiento.

\section{Ser y no parecer}

Una de las competencias del Orientador supone el desarrollo de un principio deontológico infundado en la autonomía que implica saber cómo actuar en situaciones éticas.

En base a lo anterior, se destaca la importancia de que la Orientación es un proceso de integración de habilidades y competencias de los seres humanos, pues al contrario de lo que han expresado algunos de los sectores más reaccionarios de nuestro sistema educativo, la Orientación es un derecho de los estudiantes y debe ser considerado como un factor cualitativo en los procesos de innovación en la educación 
Asimismo Miller (1971), precisó la Orientación como el proceso por el que se ayuda a los individuos a lograr la autocomprensión y autodirección necesarias para conseguir el máximo ajuste a la escuela, al hogar y a la comunidad.

Igualmente, Crites (1974) consideró que la Orientación era el proceso o programa de asistencia concebido para ayudar al individuo a elegir o adaptarse a una profesión. Y más aún, Knapp (1986), quien explicaba que este proceso se trataba de ayudar al desarrollo y formación de la personalidad de los escolares, siendo el primer objetivo de la Orientación, cuyo proceso influye en el valor de las habilidades, conocimientos, conceptos y aptitudes que aquellos adquieren.

A la vez, uno de los principios básicos de la Orientación es la prevención (Repetto, 2002). Por tanto, las intervenciones han de programarse y responder a una forma de trabajo planificada y sistemática, coherente con el contexto y la especificidad de los entornos, anticipándose, en la medida de lo posible a futuros problemas que puedan surgir, para que éstos no acontezcan o minimizar en lo posible sus efectos.

Ahora bien, Calonge (2004) señala "la Orientación como una disciplina auxiliar de la Educación debe corresponderse con la misma; considerar los objetivos que ella halla trazado, y colaborar en el logro de los mismos". (p.150). Lo expresado conlleva a proponer lineamientos para la Orientación que concuerden con lo que persigue la Educación; como por ejemplo, desarrollar nuevas prácticas en concordancia con la actual misión de la educación encausada hacia el sujeto en su contexto, en la construcción de sus proyectos de vida y desarrollo socio-personal en concordancia con las actuales exigencias sociales.

A tal efecto, la mayoría de las definiciones de Orientación incluyen la palabra ayuda como rasgo definitorio. Conviene insistir en que la Orientación es un proceso continuo que va más allá de la simple ayuda, que debe ser considerada como parte integrante del proceso educativo, que implica a todos los educadores y que debe llegar a todas las personas, en todos sus aspectos de desarrollo personal y durante todo el ciclo vital.

Según las circunstancias, la Orientación puede atender preferentemente a algunos aspectos en particular: educativos, vocacionales, personales, etc. (áreas de intervención); pero lo que da identidad a la Orientación es la integración de todos los aspectos en una unidad de acción coordinada. Se concibe entonces la Orientación como una intervención para lograr 
unos objetivos determinados enfocados preferentemente hacia la prevención, el desarrollo humano y la intervención social.

A razón de lo expuesto, la demandante realidad venezolana de alto riesgo social, aporta un marco explicativo y refuerza la necesidad de presencia de los Orientadores, no sólo en la escuela, sino también en diversos espacios de educación no formal, en los cuales puede responder a necesidades individuales y sociales, como promotores y educadores en bienestar social y desarrollo humano, dado que el hombre es un ser de naturaleza social, que llega a ser humano justamente por la incorporación y organización de las experiencias con los demás individuos en un espacio sociocultural determinado.

Por lo tanto, desde sus inicios, hasta hoy, la práctica de la Orientación se ha fundamentado en explicaciones psicológicas del comportamiento humano, centrándose en Teorías de la Personalidad y del Aprendizaje. Siendo necesario pero no suficiente, dado que el hombre es un ser de naturaleza social que llega a ser humano precisamente por la incorporación de las experiencias que se da con la interrelación con su medio circundante. En los actuales momentos, la Orientación no solo es responsable de los procesos psicológicos sino sociales, pudiendo considerar al sujeto desde la perspectiva biopsicosocial-cultural y comunitaria.

Así pues, el Orientador se encuentra en un contexto de rápidas transformaciones sociales, cambios globales que afectan en el espacio local, modificando, influyendo, distorsionando el campo educativo y la realidad concreta en la que desarrolla su práctica profesional. Por tal motivo, la Orientación se ha visto en la tarea obligada de adaptarse a estos cambios para poder llevar a cabo el proceso de abordaje adaptado a una realidad en constante cambio, han sido pues las investigaciones en esta área que han permitido avanzar teóricamente de la mano con las nuevas exigencias.

De acuerdo con Casado (2010), la situación de crisis de la Orientación en los momentos actuales está dominada por una gran paradoja, la cual se expresa en el hecho de que si bien hay consenso en justificar la práctica de la Orientación por las necesidades individuales y sociales que concurren en el marco de las instituciones educativas, resulta, empero, que a medida en que las necesidades-carencia han ido en aumento y, en consecuencia se ha incrementado la discrepancia entre lo dado y lo deseable, las necesidades-aspiración, la Orientación se ha ido debilitando. 
Al respecto, este dinamismo inherente a la profesión, paralelo a los cambios sociales que se están produciendo, ha encontrado tradicionalmente dificultades para implementarse, una de ellas se derivan de una formación inicial enfocada en gran medida hacia una intervención de corte remedial. En muchos casos, la formación y demandas de intervención se enfocan a la resolución de problemas o dificultades inmediatas, por lo que se deja a largo plazo y no se da una respuesta a las necesidades sociales.

En la actualidad, la inquietud por una formación del Orientador acorde con las demandas sociales, está calando en numerosos estudios de diferente amplitud geográfica. Explorando en ellos las necesidades de formación que emplazan con más frecuencia los profesionales de esta carrera, de acuerdo con competencias profesionales que se perciben como necesarias.

\section{Políticas Universales}

Considerando la retórica como la base que sustenta todo nuevo conocimiento, a la luz de las esencias que emergen del fenómeno, es propicio revivir lo que considera el documento de la Declaración Universal sobre Bioética y Derechos Humanos (2005), donde establece en su alcance que las cuestiones éticas relacionadas con las ciencias de la vida aplicadas a los seres humanos debe tomar en cuenta las dimensiones sociales, jurídicas y ambientales, por consiguiente, no es una situación expresa de un área disciplinar, sino de un conjunto de abstracciones unificadas en el ser humano como el protagonista de todo proceso.

Así pues, la Declaración Universal sobre Bioética y Derechos Humanos ha señalado que tratándose de decisiones adoptadas o de prácticas ejecutadas se habrán de respetar los principios éticos, siendo uno de ellos, la Autonomía y responsabilidad individual el cual está referido a la facultad de adoptar decisiones, asumiendo la responsabilidad de éstas y respetando la autonomía de los demás, en este sentido quiere decir que para las personas que carecen de la capacidad de ejercer su autonomía, se habrán de tomar medidas especiales para proteger sus derechos e intereses.

De la misma manera, esta Declaración está enfocada en las cuestiones técnicas científicas y esboza la condición de los profesionales en el área de las ciencias, sin embargo, ha sido adoptada por diferentes autores e investigadores para justificar de su área disciplinar el comportamiento de todo profesional, aportando el mayor interés en el Consentimiento Informado, restando importancia a la condición humana en el proceso de toma de decisiones, 
el enfoque humanista y la historia de la persona involucrada en el proceso, donde influye un universo de situaciones que condicionan las acciones de las personas. Es la teoría de Rogers que establece el proceso del darse cuenta en su enfoque humanista donde el ser actúa incluso desde el inconsciente. Es aquí donde se quiebra el interés superior por el respeto a la persona a ser autónoma, es olvidar que en el proceso existe la condición humana, siendo desplazado por un proceso estático para salvaguardar la postura profesional. Entonces ¿Quién Orienta?, ¿Desde dónde lo hace?

\section{Dinámica Adaptativa de la Norma}

Cada área profesional ha asumido esta Declaración como insigne responsable del actuar ético, y la ha adecuado a su formación profesional, quienes tuvieron la oportunidad de adecuarla a su sistema curricular posterior al 2005 tienen un basamento más ideado de cómo es el actuar del profesional. Sin embargo, hoy día existen profesionales anteriores a la fecha mencionada que no atravesaron por esta transición, y que además se perfilan en instituciones en todos los niveles educativos, algunos habrán tenido la oportunidad de estar en formación continua, así como existen gran cantidad de personas que se aferraron a su campo de trabajo y se quedaron con la formación recibida desde el plantel a través de la experiencia de los alumnos y allí radica también la esencia de este fenómeno. Es en la formación donde se halla la respuesta a la problemática de porque hoy día el accionar de los Orientadores no responde a un comportamiento unificado, siendo objeto de críticas, donde además entra en tela de juicio su praxis.

De la misma manera, el Ministerio del Poder Popular para la Ciencia, Tecnología e Industrias Intermedias, impulsa la aplicabilidad de los fundamentos Bioéticos como bases para la construcción de un modelo de desarrollo sustentable orientado a la Suprema Felicidad Social, considerando la Ética para la Vida, o Bioética como aquella que desarrolla los valores y principios derivados del respeto a la vida en todas sus formas, considerando el derecho de todos y todas a nacer y vivir en una sociedad justa, equitativa y en paz, y en un ambiente ecológicamente equilibrado.

Así pues, considerando que hasta el momento los documentos existentes han pretendido hacer uso de este principio y adecuarlo de acuerdo a diferentes necesidades, queda en evidencia que su estructura conceptual es de fácil manipulación y de libre interpretación, 
tomando sólo aquello para lo cual puede ser aplicable, dejando a un lado la esencia de la intervención de la condición humana.

\section{La Autonomía como principio universal}

Freire (1969), consideraba que la autonomía era una libertad de elección, una capacidad del ser humano para dirigir su independencia conscientemente, es decir, decidir responsablemente de sus acciones, viendo la autonomía como una conquista constante y un proceso de construcción de cada sujeto.

Para Paulo Freire, la idea de autonomía se vincula a la naturaleza del ser humano por ser más, una eterna búsqueda consciente del proceso de ser y estar en el mundo, conquistando una libertad que no lo aprisione sino que lo libere, tal como lo detallo en sus investigaciones, con especial interés el de la "Pedagogía del Oprimido", lo que se traduce en que se puede ser libre sin necesidad de oprimir a otros que difieran de nuestras necesidades.

En opinión de Mesquita (2002), el educador tiene un papel fundamental como mediador entre el educando y el acceso a la información, más allá de trasmitir conocimientos, debe estar atento al tipo de conocimiento, cómo y en qué momento introducirlo, ya que si no queda claro cuál es el rol del educador, esto no contribuirá a que el educando ejerza su autonomía, visto desde la praxis del Orientador quién de primera mano es Educador, este debe cuidar de su rol durante todo el proceso y así garantizar se cumpla de forma consciente el principio de autonomía "en el otro".

Nótese que en el principio de autonomía exige el respeto de la capacidad de autodeterminación de las personas, que deben tener el derecho de decidir por sí mismos si aceptan o rechazan un determinado tratamiento, lo que supone entonces de la exclusión de un estudio que pueda llevarse a cabo contra la voluntad del individuo o en base al engaño o a cualquier forma de coacción.

Por lo que la tarea del profesional se trata de acompañar a éste a descubrir y a decidir qué es lo que le parece más beneficioso para sí mismo, en función de sus circunstancias personales que sólo es conocida por la persona. La tarea del Orientador es ofrecer a la persona un punto de partida desde su perspectiva profesional, con conocimientos y experiencias, lo que estima es la decisión más acertada, iniciando entonces un diálogo donde el intercambio mutuo de información es clave y culmina cuando la persona decida qué acción acepta o rechaza. 
A partir de ahí se inicia un proceso dialógico, donde el intercambio mutuo de información tiene una importancia clave, que culmina cuando la persona decide en forma autónoma qué opción diagnóstica o terapéutica acepta y cuál rechaza, se trata entonces de encontrar un equilibrio razonable entre los imperativos de beneficencia y de autonomía. Por tanto, la autonomía no funciona en el vacío, sino que se ejerce dentro de la estructura propia de la condición humana y es allí donde se desencadena la diatriba entre el ser y el deber ser.

Siendo el principio de autonomía la potestad que tiene toda persona para tomar decisiones en torno a su vida personal, tratándose de un ser racional y consciente, con capacidad de discernimiento para orientar sus acciones y objetar aquellas instrucciones y mandatos contrarios a su conciencia, se ha utilizado este principio y el conjunto de ellos sólo a hechos científicos o formas de probanza en las investigaciones a través de lo que se conoce como el Consentimiento Informado, pero el principio de autonomía va más allá de ello y es la transformación que se pretende a través de esta hermeneusis.

\section{La Praxis del Orientador}

Específicamente en la carrera profesional de la Orientación, se sigue considerando como propio el Código de Ética del Orientador (2001), descrito cuatro años antes de la disposición expuesta por la Declaración de los Derechos Humanos, discutido y fundamentado en ese momento histórico como lo nuevo, lo correcto y lo aceptado; pero la vida está en constante movimiento, la sociedad ha ido cambiando con el acontecer, la tecnología ha desarrollado nuevos métodos de investigación y tecnología que nos pudiera acercar a nuevos modelos en Orientación y nuevas formas de considerar el accionar ético del Orientador con las exigencias del medio circundante. Así pues, 18 años después, el Código de Ética del Orientador, sigue siendo usado como manifiesto y garantía fáctica del ser y hacer de este profesional. Esto también debe ser transformado, actualizado y divulgado como una imperiosa necesidad para garantizar el correcto desempeño del Orientador en las áreas de formación para la cual ha sido preparado. Por tanto, una vez reflexionado acerca de las esencias que emergen del principio de autonomía deontológica, se hace necesario transformarlo.

\section{Deontología, Ética y Moral en la praxis del Orientador}

El principio de autonomía tiene su respaldo en la Ética que va más allá de la Deontología y se ocupa de aspectos que son necesario para dar respuestas necesarias, teniendo 
un gran compromiso en el estado de conciencia de la persona, su instinto, su formación moral, y la experiencia que ha de indicarle el sentido de las cosas correctas, y la lucha de lo que se puede y de lo que se debe, siendo estos aspectos estrictamente personales que difieren de profesional, dado que admite tantas visiones diferentes como personas. Pueden ser propuestas mediante códigos que se podrán aceptar o no pero no impuestas como normas o reglas.

Al respecto, Quintana (2016), expresa que las profesiones se regulan a sí mismas por diversos mecanismos de control; en contraste, los oficios son regulados desde fuera. La ética profesional no es una teoría general ni una ética sistemática sino una normativa especial, que establece preceptos y denegaciones, consejos y reprobaciones, que reflejan el consenso deontológico que ha de regular la actividad de todos, por tanto, los códigos deontológicos buscan regular la forma de actuar de los profesionales en el ejercicio de sus funciones.

Por consiguiente, es necesario que tanto los principios por los cuales se rigen las normas y los códigos deontológicos estén en constante actualización, haciendo uso de terminología más modernas, donde puedan ser incorporados los aspectos éticos que surgen de las socializaciones y encuentros de las comunidades científicas, donde puedan darse cuenta que los cambios en las condiciones de trabajo, las exigencias sociales, los nuevos hitos políticos; las demandas comerciales, el mejoramiento continuo; la globalización, el auge de la economía, la liberación de mercados internacionales; el desarrollo tecnológico y los servicios; repercuten en el contexto que rodea a la práctica de un profesional y por tanto influyen sobre manera en las normas profesionales y sobre la ética de quienes la practican, por ello, a través de esta investigación, ha quedado en evidencia que desde el 2005 hasta la actualidad, los cambios han sido enormes, y las comunidades y sociedades científicas se han quedado detenidas en un principio, específicamente el de autonomía, que respondía a necesidades diferentes a las de ahora, 13 años después.

Si bien es cierto, el cumplimiento del deber ha sido demostrado que es una cuestión personal, lo que quiere decir que lo que haga un profesional bien o mal afecta a los demás y pone en paréntesis la actuación de este personal en su campo de trabajo y condiciona el de los demás. Por ello, se hace notable que en muchos estratos se ha visto la figura del Orientador, como aquella que va en contra de su quehacer en la práctica diaria, y era la pregunta repetitiva ¿Qué Orienta? Y ¿Desde dónde lo hace? Los Orientadores desde su amplio sentido de la congruencia están en el deber de hacer lo correcto para el otro, es una acción desde la 
alteridad, mirar la verdad desde otro y coordinar la técnica correcta del darse cuenta sin inmiscuirse en el plano profesional, ese es el verdadero principio de autonomía, dado que el actual está generalizado y precozmente ha sido utilizado para fines de soportes de investigación, se presta para determinar diferentes objeciones con respecto a cuál debe ser el uso correcto del mismo.

\section{Metamorfosis del Principio de Autonomía deontológica para la praxis del Orientador}

Hoy por hoy es necesario dar a conocer que el principio de autonomía ha evolucionado conforme a las necesidades de la sociedad actual, debe ser reformado y acuñado por todas las profesiones en el respeto al debido proceso del otro, en garantía de su permanencia en el transcurrir de su atención, no es superfluo recordar que no es una autoridad moral quien prescribe los comportamientos, las normas deontológicas son establecidas por los profesionales mismos, sobre la base de aquello que favorece la profesión y aquello que la daña.

El resurgimiento y la transformación producto de esta hermeneusis que converge en el principio de autonomía propiamente deontológica para el enriquecimiento y fortalecimiento de la Orientación y en lo sucesivo, del Orientador, se muestra a continuación de lo que se considera su nacimiento y como ha ido evolucionando en el marco del tiempo, especificando concretamente los puntos en los que la Orientación ha surgido:

Figura $\mathrm{N}^{\circ} 1$. Metamorfosis del Principio de Autonomía deontológica.

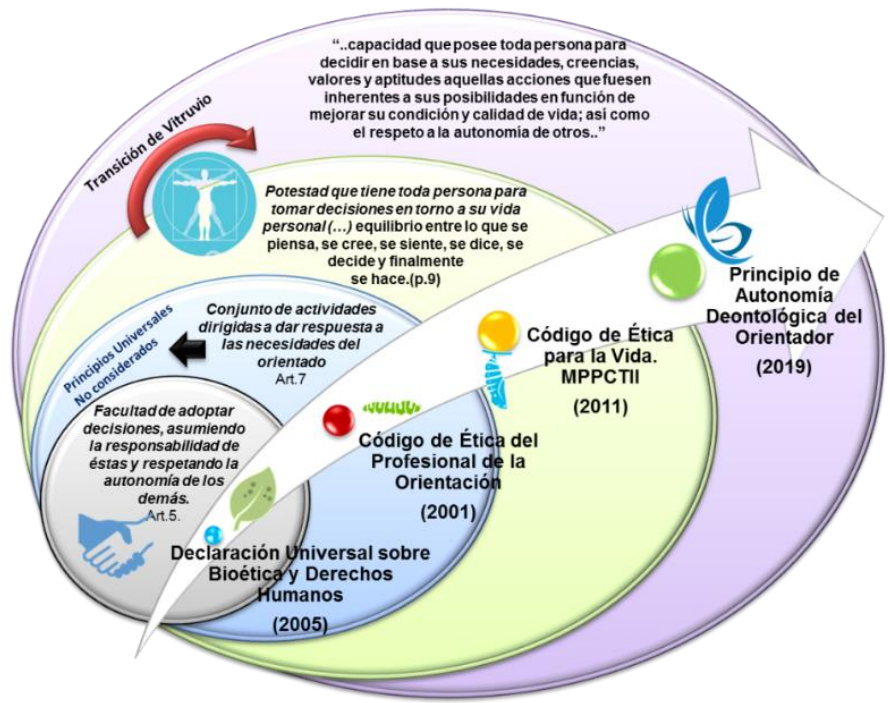

Fuente: Elaboración propia 
En primera instancia se considera la Declaración Universal sobre Bioética y Derechos Humanos declamada en 2005 producto de la preservación de la vida en toda su extensión y el respeto a otro en el pleno ejercicio de sus facultades biológicas. Expresamente el principio de autonomía es considerado como aquella facultad que tiene una persona para tomar decisiones y respetar la de terceros.

En el siguiente hilo histórico, he considerado el Código de Ética del Profesional de la Orientación por ser documento que considera el cúmulo de normas que rigen la carrera del Orientador desde el punto de vista ético; sin embargo, los principios bajo los cuales fue creado no consideran los principios universales decretados por la UNESCO en 2005, de igual forma no se aprecia el sustento teórico de los mismos y carece de sustento legal, que de igual manera, por la fecha que posee, muchas leyes han perdido vigencia. Las causas que pudieran justificar el desfase de este Ordenamiento plenamente pensado en Asamblea Nacional de Orientadores y repensado por la comunidad científica del momento, pueden ser diversas. La realidad de hoy día es que el sustento por el cual los Orientadores lo aprehenden como Código de Ético carece de la estructura Deontológica que exigen los parámetros Universales plenamente establecidos.

Es importante denotar que no se está obviando las investigaciones, grandes avances o estudios de la comunidad científica en Orientación, pero la consideración del Código de Ética del Profesional del Orientación sobre las posibles investigaciones o literaturas en ética, responde a que este es un documento público, ampliamente reconocido y acuñado por los Orientadores como la Base del Ejercicio Profesional.

En la siguiente escala de evolución se ha considerado el Código de ética para la Vida del Ministerio del Poder Popular para Ciencia, Tecnología e Industrias (2011) que establece los lineamientos filosóficos básicos y las normas que de ellos se derivan, estimulando la reflexión y contribuyendo al desarrollo de una conciencia bioética en los espacios para la ciencia, la tecnología y la producción; pero que obvia la presencia del otro y sobrepone el sí mismo por encima de cualquier situación contextual, dando importancia a la ciencia y la tecnología como principal valor de la preservación de la vida. Ofreciendo una imagen de un ser humano completo que es capaz de tomar decisiones en el pleno equilibrio de todo su ser. Por ello, he considerado antes del proceso de metamorfosis en su etapa final, lo que postulo como la "Transición de Vitruvio" para contraponer mi postura con la establecida. 
La razón por la cual he considerado la obra magnífica de Leonardo Da Vinci adaptada en 1490 que lleva por nombre "El Hombre de Vitruvio", y que ha sido considerado durante siglos como modelo de perfección en oficios y obras de arte y que en efecto, arquitectónicamente lo es. Es porque demuestra que el hombre encaja en el cuadrado y el círculo del dibujo, y que pese a que cambia la posición de brazos y piernas, el ombligo permanece en el mismo sitio. El Hombre de Vitruvio históricamente debe su nombre a Marco Vitruvio, un arquitecto romano del siglo I antes de Cristo, que trabajó para Julio César. Y fijó unas proporciones matemáticas para definir al hombre perfecto y que luego Leonardo Da Vinci aplicó estas fórmulas retocadas por él para dibujar su famoso Canon de las Proporciones Humanas.

Al respecto se debe a que, de acuerdo al Código de ética para la Vida mencionado específicamente en el principio de autonomía - considera que la toma de decisión debe darse en el pleno ejercicio de todas las facultades y la realidad de la Orientación es que durante cualquier proceso que esté experimentando, no toda persona se encuentra en el pleno ejercicio de sus funciones psíquicas, mentales, emocionales o físicas y es la misma observación realizada a la Pirámide de las Necesidades de Abraham Maslow en 1943, lo que queda preguntar. ¿Hoy por hoy es necesario atravesar todas las etapas para sentirse auto- realizado? Teóricamente, otros investigadores han ampliado este punto. Pero el espacio de convergencia en la investigación se debe a que este Código expresamente obvia el factor humano en su esencia como el ser sujeto a cambios y condicionados del mundo exterior.

Así pues, llegando al punto de enfoque de esta Tesis, presentamos el siguiente postulado como lo que considero el Principio de Autonomía Deontológica del Orientador:

Es la capacidad que posee toda persona para decidir en base a sus necesidades, creencias, valores y aptitudes aquellas acciones que fuesen inherentes a sus posibilidades en función de mejorar su condición y calidad de vida; así como el respeto a la autonomía de otros y el reconocimiento a la dignidad de la persona como derecho inalienable, expresado en total aceptación a la integridad física y psicológica en conocimiento de su forma de vida.

En caso de aquellas personas con carencias demostrables que inhiban esta toma decisión, se desarrollaran medidas alternas que puedan motivar a la persona a considerar que es lo más conveniente. Bajo ninguna condición el Orientador a cargo deberá sugerir las vías alternativas para condicionar la decisión de la persona. El Orientador, deberá agotar al máximo 
su potencial como profesional siendo capaz de dar respuestas curriculares en respeto de la condición de vida del otro.

Finalmente, considero lo que Coleridge citado por Hill (2014) llamó "la suspensión de la incredulidad", para distinguir esta investigación en el arte de la hermenéutica para recuperar el pasión por la Orientación, al considerar que ocasionalmente se olvidan las reglas del juego y se anteponen las expectativas de lectores empíricos a las expectativas del lector modelo. Al respecto, toda obra produce su propio efecto, el artista dispone de señales, indicios o sospechas para instruir al propio lector. Aunque estas señales sean ambiguas. Incluso en la interpretación, el lector modelo puede encontrar algo que el autor no sabía que había puesto. Esto no invalida que las interpretaciones deban estar justificadas por la estrategia de la obra. La obra está ahí y produce su propio efecto. La regla fundamental para afrontar la interpretación de una obra, es que el lector acepte tácitamente un pacto ficticio con el autor. (Hill, 2014)

Así pues, a sabiendas de que las decisiones relativas a las cuestiones éticas pueden tener repercusiones en los individuos, familias, grupos o comunidades y en la especie humana en su conjunto, el Orientador debe ser perspicaz en su accionar, dar respuestas a las necesidades que a su bien tenga en honor a su labor y en respeto a la de su gremio.

Respectivamente, La Orientación y la Educación son fenómenos predominantemente humanos, estos son parte de la cultura y de la sociedad, la Orientación busca contribuir, plantear y analizar las múltiples formas en la humanización se manifiesta en los tiempos actuales.

Es en el quehacer cotidiano de la Orientación donde se halla los temas relacionados con el respeto hacia el otro y hacia sí mismo, hoy por hoy denominado en esta investigación como principio de autonomía deontológica, el cual se debate en todos los contextos y que además es necesario profundizar.

Desde la ética del Orientador, es posible reflexionar acerca de cómo se establece el encuentro entre seres humanos en la acción de orientar, y como la acción del profesional en esta área logra que el sujeto ocupe un lugar en el mundo social; ardua tarea la del Orientador, al mantenerse inerte en los procesos personales y ofrecer una atención a la altura y el nivel que extrapolan los grandes pensadores. 
Reflexionar desde la ética, implica una óptica específica: la del cuestionamiento, la interpelación acerca de las acciones y también de los principios y criterios, en los que el Orientador se funda. La ética es "una reflexión teórica sobre la moral" (Lagarigue y Lebe, 1997).

Por su parte, la moral establece la acción conforme a reglas, mientras la ética cuestiona aquello que funda la moral, analiza sus fuentes, sus bases, es aquí donde el cuestionamiento interno del Orientador entra en juego, es el proceso del darse cuenta donde todo profesional debe llegar en garantía de su desempeño profesional.

En tanto, es necesario analizar de manera específica las acciones del Orientador, para razonar las situaciones que concurren entorno a la deontología y al mismo tiempo insistir en fundamentar los principios y valores de la misma. Se trata entonces de develar los principios legítimos que surgen de la práctica social en respeto genuino de la existencia del otro. Desechando todo hábito inadecuado que permite hacer válido lo que el "otro" hace y ser considerado como "modelo a seguir".

De tal manera que la ética en el campo de la Orientación Educativa, con mayor hincapié en el principio de autonomía deontológica, es una mirada sobre las acciones que el Orientador realiza, que permite justificar y fundamentar el porqué de esas acciones y no otras y de cómo éstas influyen de manera en el orientado; en plena armonía de la relación Orientador - orientado.

En opinión de Flores (2004), la ética suscita la responsabilidad de forma implicante, lo que se atribuye a problematizar los comportamientos con referencia a los valores fundamentales en un espacio donde se ejercita la libertad, se trata de una deontología inspirada en la reflexión, esto tiene como propósito final, actuar con la verdad en honra de su servicio.

De tal manera que esta investigación permitió crear una hermeneusis teórica del principio de autonomía deontológica para el Orientador del siglo XXI a través de una metamorfosis para redefinir su praxis en el medio circundante, por lo que se han develado las esencias que repercuten en su labor profesional, encontrando el vacío teórico para fundamentar su accionar desde lo establecido en la Declaración Universal sobre Bioética correspondiente al manifiesto proclamado por la UNESCO en 2005, por lo que el aporte genuino a la comunidad científica es la fundamentación teórica del Principio de Autonomía Deontológica propio del 
Orientador, que emergió de la hermeneusis y de la investigación exhaustiva de este principio y su relación con la Orientación para justificar éticamente su praxis.

Al respecto las evidencias fueron claras, hasta el momento no se había adaptado la ética fundada en el principio de autonomía específicamente, por lo que el hilo histórico permitieron demostrar que si es posible poseer el sustento ético - deontológico de la labor del Orientador, contextualizándolo además con la realidad de Latinoamérica, en plena vigencia con el nuevo siglo, por lo que este nuevo postulado acerca de la Autonomía Deontológica del Orientador, viene a reivindicar la noble y sublime tarea de este profesional como impulsor de cambios sociales desde el reconocimiento de la humanización.

\section{Referencias}

Amaya y Otros (2015). ¿Cómo aplicar losprincipios éticos? Artículo para Pagina Web Ética Psicológica. Recuperado de: http://eticapsicologica.org/index.php/documentos/articulos/item/17-como-aplicar-losprincipios-eticos

Asociación Internacional para la Orientación Educativa y Vocacional (2018).Êtica en Orientación-Expectativas y Realidad: Enfoques Teóricos, impulso internacional y experiencias prácticas porSuzanne BULTHEEL. Presidenta de la IAEVG/ AIOSP.IAEVG Hoja Informativa. 81, Junio 2018.

Ayala, R. (2007). La metodología fenomenológico-hermenéutica de Max Van Manenenel campo de lainvestigación educativa. Posibilidades y primerasexperiencias. Revista de Investigación Educativa, vol. 26, núm. 2, 2008, pp. 409-430. AsociaciónInteruniversitaria de Investigación Pedagógica. Murcia - España

Bartolomé, M. (2000). HaciadóndevalaInvestigación Educativa. Inauguracióndel curso 20002001, Facultad de Pedagogía, Universidad de Barcelona. Barcelona: DULAC Ediciones.

Calonge (2004). Fundamentos Contextuales de laOrientación Educativa. Investigación y Postgrado Online abr 2004 vol 19 No 1. p. 145 - 170. Recuperado dehttp: // www. scielo. org . ve . / scielo. php. script =Sei_arttexpid=S1316.

Camargo, X. y Leal, S. (2015). La Orientación: una praxis para laalteridad y lainclusión educativa y laboral. Revista Orientación. Facultad de Ciencias de laEducación. Universidad de Carabobo. Volumen3, No3 | Julio-Diciembre. Recuperado de: http://servicio.bc.uc.edu.ve/educacion/orienta/n3/art04.pdf

Casado, E. (2010). Leyes de servicios sociales del siglo XXI. Cáritas. Madrid.

Código de Ética del Profesional de la Educación (2001). XXI "Encuentro Nacional de Orientadores.Federación de Asociaciones Venezolanas de Orientadores (FAVO). Estado Portuguesa - Venezuela.

Crites, J. (1974): Psicología vocacional. Buenos Aires: Paidós. 
Eco, U. (1998). Cinco escritos morales. Barcelona: Lumen. Fernández Sierra, J. y Carrión Martínez, J. J. (1998). La formación de losprofesionales de laOrientación. En R. Bisquerra Alzina (coord.), Modelos de Orientacióne intervenciónpsicopedagógica. Barcelona.

Flores, M. (2004). La Reflexión Ética y laOrientación Educativa, una Relaciónpococonocida. Revista Mexicana de OrientaciónEducativa. Marzo - Junio 2004.

Freire, P. (1969). Pedagogíadel Oprimido. Contribuciones a laconcepción de Autonomíaen Mesquita D,L. (2002).

González, J (2008). La Orientacióndesde latransdisciplinariedad. Ponencia presentada enSimposio La Orientacióny La Educaciónen Valores desde una Perspectiva Transdiciplinaria. Valencia.

González, J. (2001). Perspectivas de lapráctica Orientadora enel contexto de lasnuevas dimensiones de laEducación. Revista interactivalaOrientaciónalrededordel Mundo No 4 2.001 [Revista en línea] Recuperado de: http://www.geocities.com/ Julio _ González / revista.html.

Heidegger, M. (1962). De laexperienciadel pensar y otros escritos afines. Universidad de Chile, Facultad de Filosofía, Humanidades y Educación, Departamento de Filosofía, PublicacionesEspeciales $\mathrm{N}^{\circ} 26$, Serie de Textos.

Heredia, K. (2017). Interpretación y lapuestaenpráctica de laOrientaciónenel campo laboral. Informe de Investigación. Estado Carabobo. Venezuela.

Hill, J. (2014). La completa suspensión de laincredulidad. Recuperado de: https://www.animalpolitico.com/el-congal-postapocaliptico/la-absoluta-suspension-de-laincredulidad/

Knapp, R, (1986) Orientacióndel escolar. Madrid: Morata.

Lagarigue, J. y Lebe, G. (1997). Ética o Moral. Investigación y Formación. N²4. París. INRP.

Luque, A. (2005). ¿Imprescindibles o desconectados? Sentimiento de competencia y necesidades de formación de los Orientadores enlos departamentos de Orientación. En C. Monereo y J. L. Pozo (coords.), La prácticadelasesoramiento educativo a examen. Barcelona: Graó.

Mesquita, D. (2002). El esclarecimientodel concepto de autonomía de Paulo Freire enlapráctica de Educadores sociales para niños de lacalle. Buenos Aires y México. Siglo XXI Editores. ISBN 968-23-2387-8

Miller, F. (1971): Principios y servicios de Orientaciónescolar. Madrid: MagisterioEspañol.

Ministeriodel Poder popular para laCiencia, Tecnología e Industrias Intermedias (2011). Código de Ética para la Vida. Venezuela.

Moreno, L. (2018). PrácticasenOrientación: una mirada holística. Trabajo de Investigación. UniversidaddelZulia. Maracaibo - Venezuela.

Programa de Protección y DesarrolloEstudiantil (2007). División de DesarrolloEstudiantil. Ministeriodel Poder Popular para laEducación. Caracas - Venezuela. 
Quintana, O. (2016). Deontologíadel docente, formador de formadores, enel subsistema de educaciónuniversitaria, ensu rol investigador. TesisDoctoral. UniversidadCarabobo. Estado Carabobo - Venezuela.

Repetto, T. (2002), Los equipos de apoyo externo. Modelos de Orientacióne intervenciónpsicopedagógica. Volumen1. Madrid: UNED. pp. 508-543.

\section{Rubio, M. (1992). Contratos de aprendizaje. Universidad de México: México.}

Santana, L. (2003): “El dilema del marco teórico en Orientación”. Curriculum, 2, pp. 41-58. Tenerife.

UNESCO (2005). Declaración universal sobre Bioética y Derechos Humanos. Homologada unánimemente por ciento noventa y un países en la 33. a Sesión de la Conferencia General de la UNESCO realizada en París el 19 de octubre de 2005. (p.86)

Vadillo, M. (2004). La disonancia cognitiva, o cómo el ser humano se convierte en esclavo de sí mismo. Disponible en: http://www.psicoteca.com

Van Manen, M. (2003). Investigación Educativa y Experiencia vivida. Ciencia humana para una pedagogía de la acción y de la sensibilidad. Barcelona: Idea Books.

Vilera, A. (2000). Reafirmación de la Orientación ante las transformaciones culturales: Abriendoespacios críticos (pp. 113-116). En Congreso Mundial de Orientación y Asesoramiento: 49 Puntos de Vista en Orientación. Valencia, Venezuela: Universidad de Carabobo. 